\title{
Littérature philosophique à Byzance et sa postérité à l'époque moderne
}

Conférences de l'année 2011-2012

\section{Michel Cacouros}

\section{OpenEdition}

\section{Journals}

Édition électronique

URL : https://journals.openedition.org/ashp/1445

DOI : 10.4000/ashp.1445

ISSN : 1969-6310

Éditeur

Publications de l'École Pratique des Hautes Études

Édition imprimée

Date de publication : 1 septembre 2013

Pagination : 34-39

ISSN : 0766-0677

Référence électronique

Michel Cacouros, "Littérature philosophique à Byzance et sa postérité à l'époque moderne », Annuaire de l'École pratique des hautes études (EPHE), Section des sciences historiques et philologiques [En ligne], 144 | 2013, mis en ligne le 22 octobre 2014, consulté le 05 novembre 2021. URL : http:// journals.openedition.org/ashp/1445; DOI : https://doi.org/10.4000/ashp.1445 


\title{
LITTÉRATURE PHILOSOPHIQUE À BYZANCE ET SA POSTÉRITÉ À L'ÉPOQUE MODERNE
}

\author{
Maître de conférences : M. Michel Cacouros
}

Programme de l'année 2011-2012: I. Philosophie byzantine et post-byzantine: tradition et exégèse des Analytiques d'Aristote à Byzance et dans l'après-Byzance. - II. Histoire de la culture et de l'enseignement à Byzance et dans l'après-Byzance; les disciplines du Trivium et $d u$ Quadrivium : permanences et renouvellements d'idées de Byzance à Venise et le cas de la Harmonia Definitiva Entium de mente Graecorum Doctorum (1661) de Gérasime Vlachos le Crétois (1605/7-1685).

La première partie de ce séminaire porte sur l'histoire de la philosophie byzantine et post-byzantine, plus précisément sur l'exégèse d'Aristote à Byzance et dans l'aprèsByzance jusqu'à ses derniers prolongements, au XIX ${ }^{\mathrm{e}}$ siècle. La seconde partie du séminaire porte sur la culture et l'enseignement à Byzance et dans l'après-Byzance, $\mathrm{y}$ compris ses prolongements jusqu'au XIX ${ }^{\mathrm{e}}$ siècle, en particulier sur la tradition du Trivium et du Quadrivium (Arts libéraux) pendant ces périodes.

\section{Philosophie byzantine et post-byzantine : tradition et exégèse des Analytiques d'Aristote à Byzance et dans l'après Byzance}

En 2010-2011, nous avions commencé la présentation de l'exégèse grecque des Analytiques d'Aristote; ainsi que nous l'avions signalé, notre but était « d'étudier de manière aussi complète que possible la tradition, l'exégèse et la transmission des Analytiques dans le monde grec depuis l'Antiquité jusqu'au XIX ${ }^{\mathrm{e}}$ siècle; il s'agit, surtout, de définir les traits marquants qui caractérisent, à la fois, la tradition exégétique grecque antique, puis byzantine et, enfin, post-byzantine, en en dégageant les éléments qui les caractérisent dans cette continuité pluriséculaire; en même temps, il fallait cerner les caractéristiques distinctives qui sont progressivement venues s'ajouter sur le fonds d'exégèse commun ${ }^{1}$. Dans le cadre de ce travail de longue haleine (il se concrétise sous la forme d'un ouvrage que nous parachevons sur la tradition du texte et de l'exégèse des Analytiques $^{2}$ ), nous avons également recours à la tradition de cet ouvrage et de son exégèse en arabe (nous sommes, également, arabisant), aussi bien qu'en latin.

La première année (2010-2011) a été consacrée aux questions d'ordre général que pose l'histoire du texte des Analytiques, en commençant par la genèse du texte et les

1. Citation provenant de notre rapport dans Annuaire de l'École pratique des hautes études. Section des sciences historiques et philologiques, 143 e année, 2010-2011, p. 56-61 et, en particulier, p. 57.

2. Voir notre rapport, ibidem, $142^{\mathrm{e}}$ année, 2009-2010, p. 63-70 et, en particulier, p. 63. Voir aussi notre bilan d'activités, dans le présent volume, sous la rubrique « Travaux et programmes en cours en réalisation, projets : orientations majeures ", a2, p. 35*. 
premières étapes dans sa transmission : la place des Analytiques dans le Corpus aristotelicum; l'authenticité de l'ouvrage; la répartition des Analytiques en livres et en chapitres et la désignation de cet ensemble par le terme commun «Analytiques ». Nous avions également abordé la question de la transmission de ce texte, dans la tradition manuscrite (présentation des témoins les plus importants et des caractéristiques de cette tradition, grâce à la consultation de plusieurs témoins de ce texte). En troisième lieu, nous nous étions penché, de manière plus particulière, sur les Premiers Analytiques; nous avions relevé les lieux exégétiques majeurs qui caractérisent l'exégèse de ce livre d'Aristote dans la tradition grecque (grecque antique, byzantine, post-byzantine), la tradition arabe et la tradition latine.

L'année 2011-2012 a porté, elle, comme il avait été prévu, sur l'histoire du texte des Seconds Analytiques, livre I, et de l'exégèse qui lui a été consacrée dans les traditions grecque ancienne, byzantine et post-byzantine, arabe et latine (l'année 20122013 devra porter sur le livre II des Seconds Analytiques). Le cours comportait deux parties, une première, qui était d'ordre général, et une seconde, qui portait sur des passages aristotéliciens que nous avions choisis. Dans le cadre de la première approche, nous avions abordé les questions de l'organisation du livre I des Seconds Analytiques; de sa transmission et de l'exégèse qui lui a été consacrée dans les traditions évoquées. De manière plus particulière pour le domaine grec (grec ancien, byzantin et post-byzantin), nous avons procédé à l'énumération des textes exégétiques existants (édités et inédits), sans oublier les schémas syllogistiques. La deuxième partie a été consacrée aux passages du livre I que nous avions jugés les plus intéressants (11 passages au total, d'étendue variable, allant d'un paragraphe à une page et demie). Ces passages ont été examinés à la lumière de la tradition manuscrite du texte d'Aristote en grec (divergences entre les différents manuscrits), de la tradition exégétique en grec, en arabe et en latin (comparaison des passages parallèles).

Ajoutons que, dans le cadre de notre examen, nous avons également utilisé la traduction conservée du texte aristotélicien en arabe et les traductions de ce texte en latin (soit à partir du grec, soit à partir du latin). Cette traduction en arabe a été réalisée avant 940 apr. J.-C. par Abū Bišr Mattā ibn Yūnus et elle a été établie à partir de la traduction en syriaque d'Ishāa Ibn Hunayn ${ }^{1}$. Parmi les traductions latines médiévales,

1. L'attribution en question et les autres précisions figurent dans le colophon du manuscrit utilisé par l'éditeur 'A. Badāwī; il s'agit du manuscrit bien connu Paris. ar. 2346. Le texte de cette traduction a été édité avec les autres traductions de l'Organon comprises dans le même témoin dans Manțiq Arisțū, éd. 'A. Badāwī, en 3 vol., Le Caire, 1948-1952, Dirāsāt Islamiyya 7 (réimpr. Beyrouth - Koweit, 1980), vol. II, p. 329-426 pour les Seconds Analytiques, livre I. Voir F. E. Peters, Aristoteles Arabus. The Oriental Translations and Commentaries of the Aristotelian Corpus, Leyde, 1968 (New York University. Department of Classics. Monographs in Mediterranean Antiquity), p. 17-20; H. Hugonnard-Roche, « Les traductions du grec au syriaque et du syriaque à l'arabe (à propos de l'Organon d'Aristote) », dans Rencontres de cultures dans la philosophie médiévale, Louvain-la-Neuve - Cassino, 1990 (Publications de l'Institut d'études médiévales, Rencontres de philosophie médiévale, université catholique de Louvain - Università degli studi di Cassino), p. 131-147 et, en particulier, p. 143, 146-147; Sancti Thomae de Aquino, Opera omnia, t. I* 2. Expositio libri posteriorum, editio altera retractata cura et studio fratrum praedicatorum, éd. R. A. Gauthier, Rome - Paris, 1989, p. 55*; M. Cacouros, « Exégèse grecque et problèmes éditoriaux dans le commentaire moyen d'Averroès aux Seconds Analytiques (livre II, chap. 1-10)», dans La lumière de l'intellect. La pensée scientifique et philosophique d'Averroès dans 
deux ont été effectuées à partir du grec : celles de Jacques de Venise (première moitié $\mathrm{du} \mathrm{XII}^{\mathrm{e}}$ siècle $)^{1}$ et d'un prénommé Jean $\left(\mathrm{XII}^{\mathrm{e}}\right.$ siècle); une troisième a été réalisée par Gérard de Crémone ( ca 1114-1187), à partir d'une version en arabe ${ }^{2}$. En dernier lieu, une recension de la traduction de Jacques de Venise a été effectuée en moins d'un siècle plus tard, vers 1270, par Guillaume de Moerbeke ( $c a 1215-1286)^{3}$. Les quatre traductions médiévales en latin ont été éditées dans le cadre de l'Aristoteles Latinus ${ }^{4}$. Nous avons également utilisé des traductions des Analytiques effectuées à la Renaissance. L'utilité des traductions en arabe et en latin consiste au fait qu'elles comportent, parfois au même titre que les exégèses correspondantes, des éléments interprétatifs : ce phénomène se produit car, parfois, au lieu de fournir une traduction à proprement parler, le traducteur interprète certains passages, en introduisant des éléments exégétiques qui n'existaient pas dans le texte d'origine.

Dans le cadre de l'examen de l'exégèse consacrée aux passages choisis du livre I des Seconds Analytiques, le constat le plus important était, à nos yeux, le suivant : l'exégèse de ce livre a été fortement influencée par celle au livre II; plus précisément, elle a été influencée de manière nette (y compris au niveau de la terminologie) par la division des quatre enquêtes aristotéliciennes, suivant l'exégèse, en enquêtes « simples » et en enquêtes « complexes » ${ }^{5}$ (d'après les Seconds Analytiques, livre II, $\S 1,89 \mathrm{~b} 22$ - $\$ 2,90 \mathrm{a} 6$; ce trait exégétique sera ultérieurement élaboré dans le cadre de l'examen du livre II). Cette influence ne caractérise pas seulement l'exégèse (grecque, arabe et latine, toutes ayant été passées en revue), mais elle est aussi visible dans les traductions du texte aristotélicien du livre I en arabe et en latin. Ainsi, elle est présente

son temps, éd. A. Hasnawi, Louvain, Peeters, 2011 (Ancient and Classical Sciences and Philosophy), p. 213-243 et, en particulier, p. 220 et n. 29-31.

1. Sur Jacques de Venise, qui séjourna à Constantinople et connaissait le grec, voir la bibliographie que nous avons réunie dans notre contribution « Théodore Prodrome, Robert Grosseteste, Jacques de Venise et l'histoire d'une erreur interprétative dans l'exégèse des Seconds Analytiques II, 1-2 », dans Actes du symposium de philosophie byzantine et latine, Athènes, 11-14 novembre 1993, publiés dans Cahiers de l'Institut du Moyen Âge grec et latin, 66 (1996), p. 135-155.

2. Voir Analytica posteriora, éd. L. Minio-Paluello et B. G. Dod, Bruges - Paris, 1968 (Corpus Philosophorum medii aevi, Aristoteles Latinus), t. IV, 1-4, p. LII-LXXv; cf. B. G. Dod, s. v. Aristoteles Latinus, dans The Cambridge History of Medieval Philosophy from the rediscovery of Aristotle to the disintigration of scholasticism (1100-1600), éd. N. Kretzmann, A. Kenny, J. Pinborg, éd. associé E. Stump, Cambridge, 1982, p. 45-79 et, en particulier, p. 58 et 75; Robertus Grosseteste, Commentarius in Posteriorum Analyticorm libros, éd. P. Rossi, Florence, 1981 (Corpus philosophorum Medii Aevi, Testi e Studi), p. 13-14; M. Cacouros, « Théodore Prodrome, Robert Grosseteste, Jacques de Venise », p. 139; Idem, « Exégèse grecque et problèmes éditoriaux dans le commentaire moyen d'Averroès aux Seconds Analytiques », p. 218.

3. Analytica posteriora, éd. L. Minio-Paluello et B. G. Dod, p. LXXV-LXXXIII ; cf. B. G. Dod, s. v. Aristoteles Latinus, p. 75 (datation de la traduction vers 1269 ou plus tôt); J. Brams, « Guillaume de Moerbeke et Aristote», dans Rencontres de cultures dans la philosophie médiévale, p. 316-336 et, en particulier, p. 319 (datation vers 1270); M. Cacouros, « Théodore Prodrome, Robert Grosseteste, Jacques de Venise », p. 139-140; Idem, «Exégèse grecque et problèmes éditoriaux dans le commentaire moyen d'Averroès aux Seconds Analytiques », p. 218.

4. Analytica posteriora, éd. L. Minio-Paluello et B. G. Dod, voir supra, n. 2.

5. En ce qui concerne la terminologie de cette bipartition, nous renvoyons à présent à l'étude faite dans notre contribution : « Exégèse grecque et problèmes éditoriaux dans le commentaire moyen d'Averroès aux Seconds Analytiques (livre II, chap. 1-10) », p. 224-231. 
dans la traduction conservée (en arabe) du livre I par Abū Bišr Mattā ibn Yūnus et, par la suite, dans celle de Gérard de Crémone. À ce titre, elle repose sur l'existence d'un trait exégétique d'origine grecque qui, lors de la transmission de la philosophie grecque au monde arabe $\left(\mathrm{IX}^{\mathrm{e}}-\mathrm{X}^{\mathrm{e}}\right.$ siècles), a été introduit au sein de l'exégèse arabe; puis, dans le cadre du mouvement de traduction de la littérature philosophique et scientifique arabe en latin aux $\mathrm{XII}^{\mathrm{e}}$-XIIIe siècles, ce trait exégétique a été transmis en Occident et a été développé dans l'exégèse latine.

\section{Histoire de la culture et de l'enseignement à Byzance et dans l'après- Byzance; les disciplines $d u$ Trivium et du Quadrivium : permanences et renouvellements d'idées de Byzance à Venise et le cas de la Harmonia Definitiva Entium de mente Graecorum Doctorum (1661) de Gérasime Vlachos le Crétois (1605/7-1685)}

La seconde partie de ce séminaire est consacrée à la culture et l'enseignement à Byzance et dans l'après-Byzance; à ce titre, elle porte sur la conception et l'organisation de l'enseignement à Byzance et dans l'après-Byzance, à partir de ses premières étapes jusqu'aux niveaux les plus élevés, sur la structure des cycles éducatifs pratiqués pendant les périodes envisagées, sur l'évolution qu'ils ont connue dans le temps, sur les matières qui y étaient enseignées et, de manière plus globale, sur l'ensemble des questions liées à ces sujets.

Il s'agit d'une approche en grande partie technique, qui, en exploitant le témoignage des sources, a comme point central la tradition du Trivium et du Quadrivium (Arts libéraux), autrement dit la tradition des disciplines littéraires et scientifiques qui constituaient la base de l'enseignement supérieur. Cette tradition est examinée pour Byzance, mais, aussi, pour les espaces géopolitiques où les Byzantins ont dû séjourner après 1453 : l'espace balkanique, mais, aussi, l'Occident, où plusieurs parmi eux se sont refugiés, en formant parfois d'importantes communautés.

En même temps, cette seconde partie de notre séminaire est conçue et envisagée de manière plus large et, pour ainsi dire, extérieure : en effet, depuis plusieurs années, nous essayons de voir la manière dont l'éducation et la culture byzantines ont généré une idéologie et un ensemble de traditions, qui ont prévalu à Byzance aussi bien que dans l'après-Byzance. Ces éléments sont directement liés à l'histoire des mentalités et aux mouvements de pensée qu'ils ont su engendrer au fil des siècles; la présence et le caractère fonctionnel de ces éléments peuvent être remarqués même après la "régénération » de la Grèce, suite à la résurrection de 1821, et la création du premier État grec; dans certains cas, ils se prolongent même davantage. Dans notre présentation, nous essayons également de suivre les permanences diachroniques et les traditions de cette idéologie byzantine, en parvenant jusqu'à ses dernières manifestations. La permanence de la tradition du Trivium et du Quadrivium (Arts libéraux) constitue, dans ce cas aussi (et sous les points de vue évoqués : histoire des mentalités et mouvements de pensée), un sujet majeur de notre séminaire.

Pendant cette année, nous avons continué l'étude de l'œuvre de Gérasime Vlachos le Crétois (1605/7-1685), devenu métropolite de Philadelphie (autrement dit, métropolite à Venise) vers la fin de ses jours. Ainsi que nous l'avions souligné l'année dernière, 
«plus de deux siècles après la chute de Constantinople, Vlachos est sans conteste possible un véritable Byzantin. Ce constat est visible, tout d'abord, dans l'approche qu'il adopte vis-à-vis des sujets abordés et qui est, comme à Byzance, cumulative, "encyclopédique" et anthologique. Ce constat est également visible dans ses goûts et choix littéraires. À ce titre, le Trivium et le Quadrivium, la philosophie et la théologie sont, comme à Byzance, les axes majeurs autour desquelles s'articule son œuvre. De plus, la philosophie est répartie, dans son œuvre, d'après les grandes divisions dont Byzance avait héritée : la logique, la physique et la métaphysique, l'éthique $»^{1}$.

Plus particulièrement, en prolongeant l'étude commencée l'année dernière, notre intérêt a porté sur le recueil de Horoi qui, composé par cet érudit et publié en 1661, porte le titre Harmonia Definitiva Entium de mente Graecorum Doctorum. En 20102011, à l'exception des problèmes liés à la biographie de Vlachos, nous avions abordé la question du genre exégétique auquel appartient cet ouvrage, à savoir les recueils de $H o r o i^{2}$. En effet, sous le titre - particulièrement énigmatique aux yeux des lecteurs d'aujourd'hui - Harmonia horistikè tôn ontôn - Harmonia Definitiva Entium (en grec et en latin dans le titre de l'ouvrage), Vlachos présente un recueil de définitions similaire à ceux que nous avions déjà examinés.

En même temps, nous avions commencé l'étude des sources variées utilisées par cet auteur. Cette année, nous avons étudié, en premier lieu, la Prothéoria (examen préliminaire, introduction) de Gérasime Vlachos, qui offre la clé d'accès à son ouvrage; dans celui-ci, Gérasime Vlachos explique les raisons qui l'ont incité a composer ce recueil; dans son exposé, il reprend pour l'essentiel la thèse byzantine sur la nécessité de l'enseignement et de l'instruction que nous avions décrite dans un autre contexte : en effet, pour les Byzantins, aussi bien que pour Gérasime Vlachos, l'instruction et la culture constituaient le seul moyen permettant à l'Homme de remédier à sa chute et à son exil du paradis ${ }^{3}$. Ainsi, signale-t-il, « le sage Prométhée, à savoir la Providence de Dieu, qui transcende la sagesse, a fait descendre du ciel le feu de la connaissance et de la science aux hommes $»{ }^{4}$.

À cette théorie byzantine sur la nécessité de l'enseignement, Gérasime Vlachos ajoute un élément significatif : ce dernier est certes, lui aussi, d'origine byzantine, mais, au lieu de provenir de la théorie byzantine sur l'enseignement, il relève d'un domaine différent, à savoir de l'exégèse byzantine d'Aristote. Plus précisément, il s'agit d'une référence aux quatre méthodes susceptibles d'être pratiquées dans le cadre de toute enquête scientifique (les éléments qui suivent sont étudiés dans la première partie

1. Citation d'après notre rapport dans Annuaire de l'École pratique des hautes études. Section des sciences historiques et philologiques, $143^{\mathrm{e}}$ année, 2010-2011, p. 60.

2. Sur ce type d'écrit, qui a retenu notre attention à plusieurs reprises, nous nous contentons de renvoyer à notre contribution « Le commentaire d'Aristote à Byzance et ses aspects peu connus : caractéristiques, tendances et perspectives » [en grec], Hypomnèma stè philosophia, 4 (2006/2), p. 155-190, et, en particulier, p. 162.

3. Voir notre contribution : «L'enseignement des disciplines littéraires (Trivium), mathématiques (Quadrivium) et de la philosophie à Byzance : conception et organisation, pratiques de l'oral et techniques de l'écrit », dans les actes du colloque historique international De l'Antiquité à nos jours : histoire et méthodes de l'enseignement, organisé par M.-O. Munier, Abbaye-École de Sorèze, 26-27 octobre 2006, publié aux Presses du centre universitaire Champollion, Albi, 2007, p. 1-83, et, en particulier, p. 14-19.

4. Traduction du passage de la Harmonia horistikè tôn ontôn-Harmonia Definitiva Entium, p. 3. 
de notre séminaire, consacrée aux Analytiques) : l'Horistique (qui repose sur l'usage des définitions, en grec horismoi); l'Apodictique (qui exploite les démonstrations, en grec apodeixeis); la Diérétique (à savoir la science des divisions, en grec diaireseis) et l'Analytique (qui repose sur l'analyse, analysis). Sur cet ensemble, l'Apodictique correspond à la véritable science, enseignée par Aristote dans les Seconds Analytiques, livre II. L'Horistique, qui procure la connaissance des définitions des êtres vivants, lui est intimement liée.

Ainsi, si le recueil des définitions proposée par Vlachos procure au lecteur une connaissance véritablement scientifique, c'est parce qu'il repose sur l'usage de la définition et, par conséquent, de manière indirecte, de la démonstration. Le terme de harmonia utilisée par Vlachos renvoie à l'équilibre établi grâce à l'usage de ces deux méthodes. Il renvoie également, de manière directe, au rétablissement de l'Homme après sa chute grâce à l'accès à la connaissance, que Dieu lui a permis d'avoir; ainsi, l'Horistique et l'Apodictique deviennent les véritables outils de la rédemption de l'Homme et de son salut. Dans ce cadre, l'usage des définitions choisies acquiert une valeur significative. En réalité, Vlachos fournit au total la définition de 172 concepts. Les définitions commencent, comme il le souligne dans sa Prothéôria, par la question de Dieu $\left(n^{\circ} 1\right)$ et finissent par une question consacrée au même sujet $\left(n^{0} 172\right)$.

Étant donné que les définitions présentées recouvrent, comme il a été signalé, sans plan apparent, les domaines de la philosophie, de la théologie, du Trivium et du Quadrivium, nous avons prolongé l'étude des sources de la Harmonia Definitiva en définissant les différents ensembles thématiques et en commençant par les définitions consacrées à la logique. En effet, dans le cadre de chaque définition, Vlachos ne se contente pas de fournir uniquement la définition concernée, mais il donne aussi les définitions annexes. Ainsi, dans le cadre de la définition du prédicat et de la prédication (Définition $\mathrm{n}^{0} 30$ ), il fournit aussi plusieurs définitions des cinq prédicables de Porphyre (genre, espèce, différence, propre, accident). Notre étude devra s'achever dans deux ans. 